\title{
ARTICLE The human lung mucosa drives differential Mycobacterium tuberculosis infection outcome in the alveolar epithelium
}

\author{
J. M. Scordo ${ }^{1,2}$, A. M. Olmo-Fontánez ${ }^{2}$, H. V. Kelley ${ }^{1,2}$, S. Sidiki ${ }^{1}$, J. Arcos ${ }^{1}$, A. Akhter ${ }^{2}$, M. D. Wewers ${ }^{3}$ and J. B. Torrelles ${ }^{1,2}$
}

Mycobacterium tuberculosis (M.tb) is deposited into the alveolus where it first encounters the alveolar lining fluid (ALF) prior contacts host cells. We demonstrated that M.tb-exposure to human ALF alters its cell surface, driving better M.tb infection control by professional phagocytes. Contrary to these findings, our results with non-professional phagocytes alveolar epithelial cells (ATs) define two distinct subsets of human ALFs; where M.tb exposure to Low (L)-ALF or High(H)-ALF results in low or high intracellular bacterial growth rates in ATs, respectively. H-ALF exposed-M.tb growth within ATs was independent of M.tb-uptake, M.tb-trafficking, and M.tb-infection induced cytotoxicity; however, it was associated with enhanced bacterial replication within LAMP-1 ${ }^{+} / \mathrm{ABCA} 1^{+}$ compartments. H-ALF exposed-M.tb infection of ATs decreased AT immune mediator production, decreased AT surface adhesion expression, and downregulated macrophage inflammatory responses. Composition analysis of H-ALF vs. L-ALF showed H-ALF with higher protein tyrosine nitration and less functional ALF-innate proteins important in M.tb pathogenesis. Replenishment of H-ALF with functional ALF-innate proteins reversed the H-ALF-M.tb growth rate to the levels observed for L-ALF-M.tb. These results indicate that dysfunctionality of innate proteins in the H-ALF phenotype promotes M.tb replication within ATs, while limiting inflammation and phagocyte activation, thus potentiating ATs as a reservoir for M.tb replication and survival.

Mucosal Immunology (2019) 12:795-804; https://doi.org/10.1038/s41385-019-0156-2

\section{INTRODUCTION}

In the early 21 st century Tuberculosis (TB) is one of the deadliest infectious diseases worldwide, killing 1 person every $21 \mathrm{~s}{ }^{1}$ TB is caused primarily by the pathogen Mycobacterium tuberculosis ( $M$. tb) and transmitted by inhalation where it is delivered into the distal lung, i.e., the alveolus. ${ }^{2}$ The early innate immune response within the alveolus may determine the course of M.tb infection. ${ }^{2,3}$ To prevent successful colonization requires a better understanding of the initial infection site, i.e., the alveolus, including soluble innate components of the lung mucosa involved in the early M.tbhost cell interactions.

The alveolus is lined with lung mucosa, composed of a surfactant lipid layer and an aqueous-hypophase called alveolar lining fluid (ALF). ALF contains innate soluble components ${ }^{2,4-8}$ with the primary role of maintaining proper pulmonary function. ${ }^{9,10}$ Several components of human ALF, including complement and surfactant proteins (SPs), have been well characterized for their importance in host immunity against $M . t b .^{4-8,11}$ Our laboratory has defined the critical role of innate ALF-hydrolases during M.tb infection. ${ }^{4-7}$ We have shown that ALF hydrolases alter the M.tb cell wall surface resulting in significantly decreased cell surface expression of two major M.tb virulence factors, mannosecapped lipoarabinomannan and trehalose dimycolate. ${ }^{7}$ These ALFinduced M.tb surface modifications enhanced the ability of human macrophages and neutrophils to kill M.tb..$^{4-7}$

Recent studies have identified alveolar epithelial cells (ATs) as being involved in M.tb infection. While we have demonstrated a role for ALF in altering M.tb-phagocyte interactions, ${ }^{4-7}$ the impact of human ALF on M.tb infection of non-professional phagocytes, such as ATs, is unknown. Here we determined that exposure of $M$. tb to different healthy human ALFs drives differential bacterial intracellular growth in ATs, which is independent of initial AT-M.tb uptake. Based on this observation, we identified a subset of human ALFs that drove a low-M.tb growth rate [Low(L)-ALF-M.tb] and another subset that drove a high-M.tb growth rate [High(H)-ALF-M. $t b$ ] within ATs. We further demonstrated that exposure of M.tb to H-ALF (H-ALF-M.tb) did not affect M.tb intracellular trafficking within ATs; however, it induced higher active M.tb replication rates within AT multivesicular bodies (MVBs) and late endosomal compartments. H-ALF-M.tb infection reduced surface adhesion expression on ATs, drove a significant reduction of chemokine and cytokine release by ATs, and induced less AT cell death. Moreover, in comparison to L-ALF-M.tb, ATs infected with H-ALF-M.tb reduced the macrophage inflammatory response, suggesting a role for ALF in modulating M.tb-host cell interactions in the alveolar space. We found that H-ALF has higher levels of protein tyrosine nitration and that $\mathrm{H}$-ALF-innate proteins are less functional in their ability to bind to M.tb. Lastly, replenishment of H-ALF with functional ALFproteins (i.e. SP-D, SP-A and complement component C3, alone or in combination) reversed the H-ALF exposed M.tb growth rate to the levels observed in ATs infected with L-ALF-M.tb. Overall these findings suggest that M.tb exposure to H-ALF and subsequent infection of ATs provide a niche for enhanced M.tb replication; and by modulating levels and function of ALF-innate proteins we can drive decreased M.tb growth in ATs, regulating infection outcome in these cells and potentially in the alveolar space.

\footnotetext{
${ }^{1}$ Department of Microbial Infection and Immunity, Center for Microbial Interface Biology, College of Medicine, The Ohio State University, Columbus, OH 43210, USA; ${ }^{2}$ Texas Biomedical Research Institute, San Antonio, TX 78229, USA and ${ }^{3}$ Department of Internal Medicine, Pulmonary, Critical Care and Sleep Medicine Division, College of Medicine, The Ohio State University, Columbus, $\mathrm{OH} 43210$, USA

Correspondence: J. B. Torrelles (jtorrelles@txbiomed.org)
}

Received: 31 August 2018 Revised: 10 February 2019 Accepted: 25 February 2019

Published online: 7 March 2019 
a
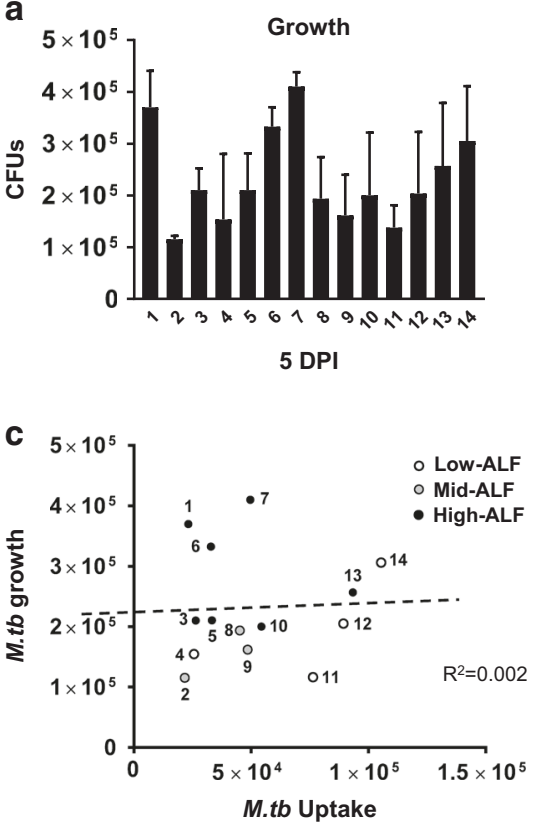

b
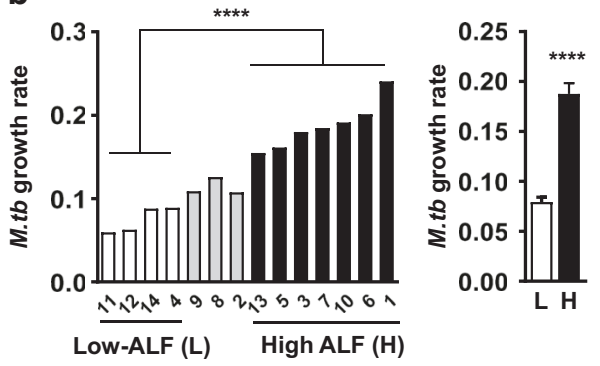

d

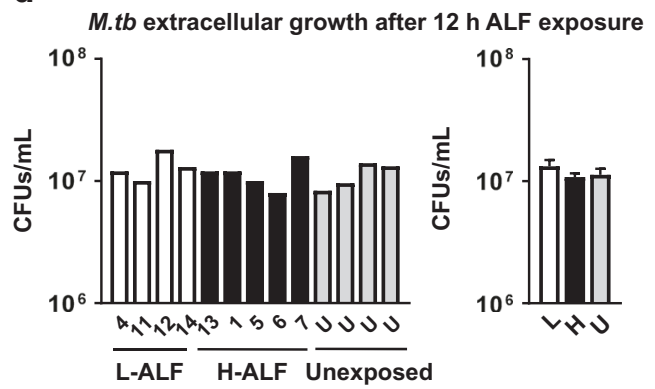

Fig. 1 Human alveolar lining fluid drives M.tb growth in ATs. a ALF-exposed M.tb intracellular growth in ATs 5 days post-infection (DPI) was assessed by colony forming units (CFUs) for determination of intracellular M.tb. Data were normalized to AT cell count. Numbers 1-14 represent ALFs, each from a different healthy human donor. Numbers between graphs correspond to the same ALF. Errors bars represent technical replicates (all ALFs were performed in replicate within the same experiment) and/or multiple experiments (for ALFs 5, 6, 9, 11, and 13). b Exposed-M.tb growth rate calculated as slope of intracellular M.tb growth throughout 5 day AT infection. Growth rate is an average value obtained from replicate experiments of $n=3$ for ALFs 11 , and $13, n=2$ for ALFs 2, 4, 5, 6, 9, 12, and 14 and for ALFs $1,3,7,8$, and $10 n=1$. L defined as Low Growth (L)-ALF-M.tb (white bars) and H defined as High Growth (H)-ALF-M.tb (black bars) with the difference in growth rate $>0.065$; Student's $t$-test L-ALF vs. High-ALF ${ }^{* * * *} p<0.0001$. c Linear regression of ALF-M.tb uptake ( $2 \mathrm{~h}$ post-infection, $\mathrm{x}$-axis) vs. ALF-M.tb intracellular growth at 5 DPI ( $y$-axis). $R^{2}=0.002$. Low-ALF (white circles), High-ALF (black circles), and mid-ALFs (grey circles). d M.tb exposed to L-ALF, H-ALF, or left unexposed was serially diluted in $7 \mathrm{H} 9$ broth and plated on $7 \mathrm{H} 11$ agar plates for determination of M.tb viability (extracellular growth) by CFUs. Shown are individual exposed-M.tb with $n=4$ L-ALF (white bars), $n=5$ H-ALF (black bars), and $n=4$ unexposed-M.tb (U, grey bars), with combined values shown in the graph on right. Freshly ALF exposed-M.tb were always used immediately for AT infections for determination of ALF exposed-M.tb growth rates in ATs

\section{RESULTS}

The human lung mucosa modulates the interaction of M.tb with ATs

In contrast to our previous findings with professional phagocytes $^{4-7}$ we observed that exposure of M.tb to human ALF drove highly variable M.tb intracellular growth in ATs (using $n=14$ different human ALFs, Fig. 1a), which did not correlate with the degree of M.tb uptake by ATs, i.e., ALF-M.tb with similar uptake (supplementary Fig S1) presented differential growth within ATs (Fig. 1a). We next defined ALF-exposed M.tb intracellular growth based on the slope of the growth curve, i.e., M.tb growth rate, during 5 days of intracellular AT infection (Fig. 1b). By this definition, we were able to statistically identify two distinct human ALF subsets: a subset of ALFs that drove low M.tb intracellular growth rate within ATs (Fig. 1b, white bars, Low(L)ALFs), and a subset that drove high M.tb intracellular growth within ATs (Fig. 1b, black bars, High(H)-ALFs). Interestingly, for $M$. tb exposed to $\mathrm{H}-\mathrm{ALF}$, we observed $2 \mathrm{X}$ the growth rate relative to L-ALF M.tb (Fig. 1b, right panel). Our linear regression analysis showed no significant correlation $\left(R^{2}=0.002\right)$ between H-ALF exposed vs. L-ALF exposed M.tb initial uptake by ATs ( $2 \mathrm{~h}$ postinfection, $2 \mathrm{~h} \mathrm{PI}$ ) and intracellular growth in ATs (5 days postinfection, 5 DPI) (Fig. 1c). We further confirmed that exposure of M.tb to either L- or Hi-ALF has no effect on bacterial viability and capability to replicate extracellularly (Fig. 1d, L-ALF-M.tb vs. HALF-M.tb vs. unexposed (U)-M.tb). Overall, these data suggest that exposure of M.tb to human ALF drives differences in M.tb intracellular growth in ATs independent of the levels of bacterial uptake.
Exposure of M.tb to H-ALF does not alter its trafficking but accelerates its replication within ATs

We next investigated if differential intracellular trafficking of Land H-ALF exposed-M.tb could explain the differences in growth rate phenotypes. Our results looking at co-localization of L- vs. $\mathrm{H}-$ ALF exposed-M.tb with the late endosomal/lysosomal marker LAMP-1, the autophagosomal marker LC3, the ATP-binding cassette $(A B C)$ lipid transporter marker of MVBs $A B C A 1^{12,13}$ and lamellar bodies (LBs) $A B C$ transporter marker $A B C A 3,{ }^{12,13}$ showed that both L-ALF and H-ALF exposed-M.tb similarly co-localized within all these compartments (Fig. 2a-f). It is plausible that individual L- and H-ALF exposed-M.tb may traffic differently; however, when plotted individually no significant differences were found among them for any of the AT intracellular markers studied (Fig S2A-D). Using the acidic probe LysoTracker, we also observed similar rates of acidification of intracellular compartments containing L- and H-ALF exposed-M.tb (Fig. $2 \mathrm{~g}$, h). These results indicate that differences observed in M.tb intracellular growth rate after being exposed to L- vs. H-ALF are not due to a differential use of the AT intracellular trafficking network.

Using a fluorescent M.tb replication reporter (SSB-GFP, smyc':: mCherry M.tb), we next determined the percentage of actively replicating L-ALF vs. H-ALF exposed-M.tb during AT infection. ${ }^{14}$ Our results indicated that $M . t b$ exposed to H-ALF had significantly increased replication ( $46 \%$ increase) within ATs (Fig. 3a, b), supporting our findings of H-ALF-M.tb having increased growth rate during AT infection.

In order to address which AT compartment H-ALF-M.tb favors to replicate, we determined co-localization of $\mathrm{H}$-ALF-exposed $\mathrm{SSB}^{+} M$. 
a
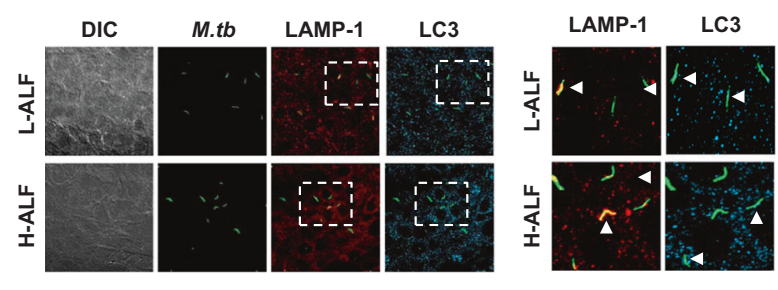

d
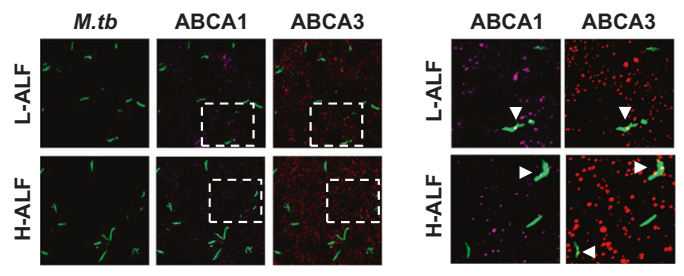

g
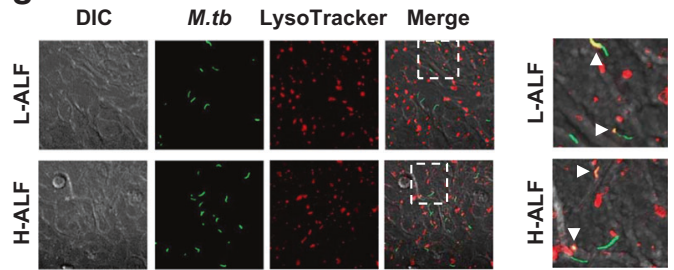

b

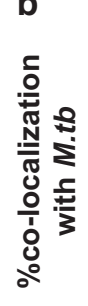

C

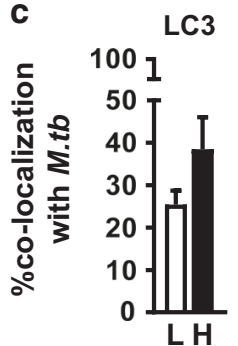

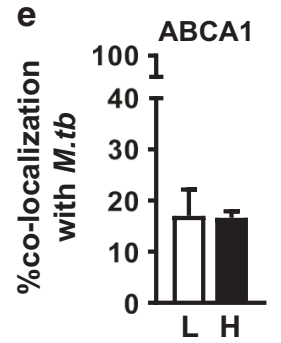

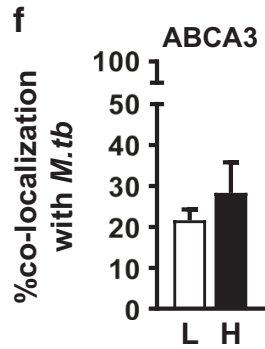

h

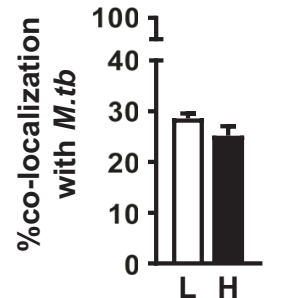

Fig. 2 L-ALF and H-ALF does not alter M.tb intracellular trafficking within ATs. GFP-M.tb co-localization with intracellular markers by confocal microscopy at 3 DPI. a Representative confocal images showing M.tb exposed to Low ALF (top panels) or High ALF (bottom panels) colocalization with LAMP-1 (red) and LC3 (light blue). The region in white dashed-line box is shown on the right with merged events indicated by white arrowheads. b Quantification of LAMP- $1^{+}$M.tb co-localization events for $n=3$ Low ALFs and $n=4$ High ALFs. c Quantification of LC $3^{+}$M.tb co-localization events for $n=2$ Low and High ALFs. d Representative confocal images of Low- (top panels) and High-ALF-M.tb (bottom panels) co-localization with ABCA1 (purple) and ABCA3 (red) at $3 \mathrm{DPI}$. The region in white dashed-line box is shown on the right with merged events indicated by white arrowheads. e Quantification of ABCA $1^{+}$M.tb co-localization events for $n=3$ Low ALFs and $n=4$ High ALFs. f Quantification of $\mathrm{ABCA}^{+}$M.tb co-localization events for $n=3$ Low and High ALFs. g Representative confocal images showing ALF-exposed M.tb (top panels, L-ALF; bottom panels, H-ALF) co-localization with LysoTracker in infected ATs at 3 DPI. White dashed-line box region is shown on the right, with merged events indicated by white arrowheads. h Quantification of LysoTracker co-localization with $n=2$ Low ALF-M.tb and $n=3$ High ALF-M.tb. All co-localization experiments were quantified by counting $>100$ events per coverslip, in replicate

tb with MVBs, LBs, autophagosomes, and late endosomes (Fig. 3c, d). Of the total replicating H-ALF-M.tb, we observed H-ALFexposed M.tb favors replication within $\mathrm{ABCA}^{+}$and $\mathrm{LAMP}-1^{+}$ compartments (Fig. 3d), suggesting that both AT MVBs and late endosomal compartments could be a niche supporting enhanced H-ALF-M.tb replication.

H-ALF exposed-M.tb infection reduces AT cell death

Published reports indicate that M.tb drives AT necrosis leading to host tissue damage and bacterial dissemination. ${ }^{15,16}$ Looking at AT cell death mechanisms, we quantified AT early apoptosis and the later stages of cell death resulting either from late apoptotic or necrotic mechanisms. ${ }^{4-6}$ We observed that H-ALF M.tb induced early and late apoptosis at similar levels to that observed in uninfected cells (Fig. 4a). We also observed H-ALF-M.tb decreased AT \% cytotoxicity (measured by $\mathrm{LDH}$ release as a marker of necrosis) relative to L-ALF-M.tb (Fig. 4b). It is important to note; however, that in both L- and H-ALF-M.tb infected ATs we observed low levels of cytotoxicity throughout the 5 days of infection (15$30 \%)$. This supports that our observed M.tb growth rate phenotypes and enhanced H-ALF M.tb replication is not a consequence of AT cell death.
H-ALF exposed-M.tb infection induces decreased AT expression of immune mediators

As the first line of defense in the alveolar space, ATs may regulate cell infiltration and activation through immune mediator production during M.tb infection. ${ }^{17}$ When we compared L-ALF-M.tb vs. HALF-M.tb, we found that H-ALF-M.tb induced significantly less interleukin-8 (IL-8) and monocyte chemoattractant protein 1 (CCL2 or MCP-1) production by ATs (Fig. 4c); however, no differences in CCL5 \& CXCL5 were observed (Supplementary Fig S3A). In addition to chemokines, ATs also produced pro-inflammatory cytokines important for M.tb control, including IFN- $\gamma$ as previously shown. ${ }^{18}$ When compared to L-ALF-M.tb, H-ALF-M.tb infected ATs induced less IFN- $\gamma$ and IL- 6 release by ATs (Fig. $4 d$ ). No differences in the production of TNF, IL-12p40, IL-10 and TGF- $\beta$ (data not shown) and expression levels for GM-CSF, MMP9, LL37 and NOS2 (supplementary Fig S3B) were observed in ATs infected with either L- or H-ALF-M.tb. We observed increased expression of IL-1 $\beta$ and decreased expression of IFN- $\beta$ genes induced by H-ALF-M.tb, but did not detect these differences at the protein level (supplementary Fig S3B and data not shown).

Interestingly, when compared to L-ALF-M.tb, ATs infected with H-ALF-M.tb had significantly decreased gene and surface 
a

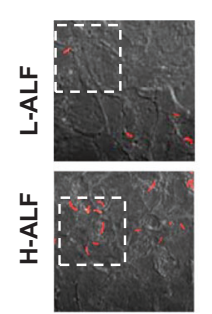

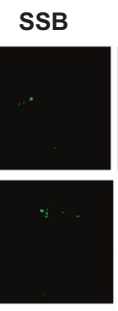
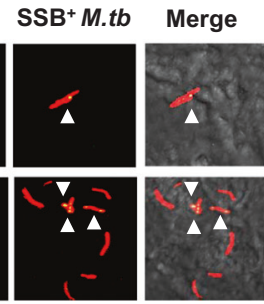

C

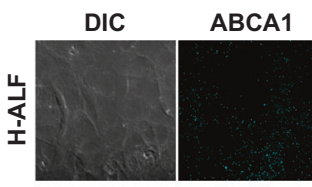

DIC

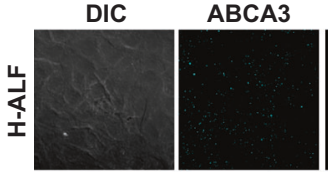

DIC

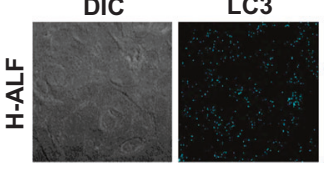

DIC

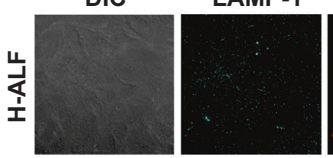

M.tb

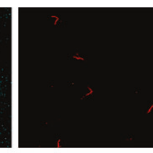

M.tb

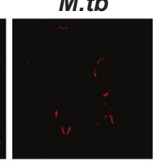

M.tb

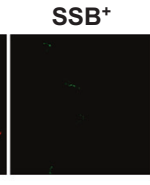

SSB $^{+}$

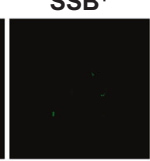

$\mathrm{SSB}^{+}$

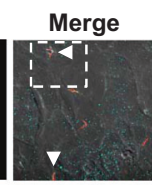

Merge

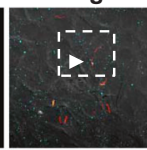

Merge

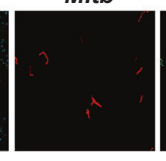

M.tb

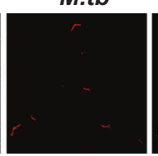

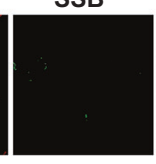

$\mathrm{SSB}^{+}$

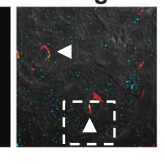

Merge
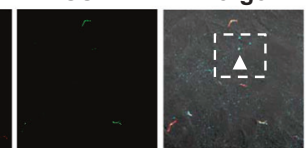

$\mathrm{ABCA1}^{+} \mathrm{SSB}^{+}$
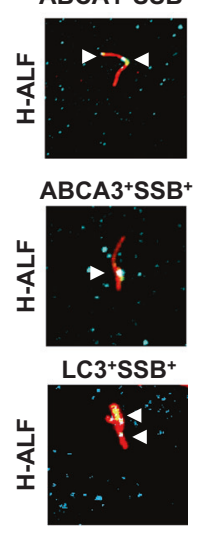

LAMP $-1^{+}{ }^{+} S^{+}$

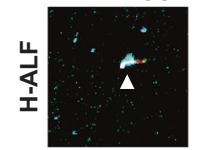

b

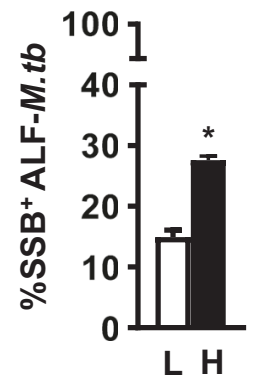

Fig. 3 High-ALF-M.tb has increased rate of replication during AT infection and favors AT MVBs and late endosomes. ATs were infected with SSB-GFP, smyc'::mCherry M.tb for 3 days and M.tb replication rate was determined by confocal microscopy. a Representative confocal images of ATs infected with Low- and High-ALF-M.tb. The region indicated by white dashed-line is shown on right (L-ALF, top panels and H-ALF, bottom panels). SSB ${ }^{+}$M.tb are indicated by white arrowheads, showing merged (yellow) foci. b Percentage of SSB ${ }^{+}$M.tb exposed to low and high ALFs at $3 \mathrm{DPI}$ was quantified by counting $>100$ events per coverslip, in replicate $(\mathrm{M} \pm \mathrm{SD}) . N=2 \mathrm{~L}$ and $2 \mathrm{H}$ ALFs; Student's $t$ test L-ALF vs. High-ALF ${ }^{*} p$ $<0.05$. c Representative confocal images of ATs infected with High-ALF-M.tb. The region indicated by white dashed-line is shown on the right. White arrows indicate SSB ${ }^{+}$M.tb co-localization with respective intracellular AT marker. d Percentage of SSB ${ }^{+} \mathrm{H}_{-} \mathrm{ALF}$ exposed M.tb co-localized with the following: ABCA1, ABCA3, LC3, and LAMP-1. Each dot represents M.tb exposed to a H-ALF in replicate, quantified by counting $>25$ events per coverslip $(\mathrm{M} \pm \mathrm{SEM})$. One-way ANOVA post-Tukey analysis; for LAMP-1 vs. ABCA3 \& LAMP-1 vs. LC3 ${ }^{*} p<0.05$

expression of the leukocyte adhesion molecule ICAM-1 (intracellular adhesion molecule 1/CD54) (Fig. 4e), and increased surface expression of CCR2 (Supplementary Fig S3C). However, no differences in AT surface expression of other markers implicated in respiratory infections, such as E-cadherin and $\mathrm{MHCl}$ were observed (Supplementary Fig S3C and D). Overall, these findings suggest that H-ALF-M.tb infection of ATs decreases their production of phagocyte-attracting chemokines and adhesion molecule surface expression without triggering a pro-inflammatory response, favoring its high growth rate within ATs.

L- and H-ALF-exposed M.tb infected ATs differentially modulate phagocyte immune responses

Published reports indicate a role for ATs in M.tb infection by modulating the function of other cell populations present in the alveolar space. ${ }^{17}$ To explore the AT-phagocyte interaction in response to L- vs. H-ALF-M.tb, we performed both direct (resting macrophages co-cultured with L- or H-ALF-M.tb infected ATs) and indirect studies (resting macrophages exposed to $0.2 \mu \mathrm{m}$-filtersterilized supernatants from L- or H-ALF-M.tb infected ATs). Our results indicate that relative to L-ALF-M.tb, H-ALF-M.tb infected ATs drove a decreased macrophage release of TNF, IL-1 $\beta$ and IL-12p40 through direct contact (Fig. 5a), and decreased TNF via indirect contact (Fig. 5b). Moreover, relative to L-ALF-M.tb, soluble mediators released from H-ALF-M.tb infected ATs reduced the surface expression of activation markers in macrophages, i.e. CD80, but did not alter the expression of a major phagocytic receptor in macrophages, the mannose receptor (MR/CD206), or HLA-DR (MHCII) (supplementary Fig S4). These findings suggest that M.tb exposed to human ALF driving high M.tb growth and active replication rate in ATs may further modulate AT-phagocyte interactions, dampening inflammatory responses that could contribute to M.tb replication and survival within the alveolar space.

High-ALF has higher levels of protein tyrosine nitration and less functional ALF-innate proteins

Based on our observation of H-ALF- vs. L-ALF-M.tb growth rate phenotypes in ATs, we chose to evaluate $\mathrm{H}$ - and L-ALF status and composition. We tested differential levels of oxidation in $\mathrm{H}$ - and $\mathrm{L}-$ ALF subsets by quantifying the presence of 3-nitrotyrosine and carbonyl residues, stable markers of NO-induced and ROS-induced protein oxidation respectively, in L- and H-ALFs. Results showed that H-ALF, when compared to L-ALF, had significantly higher levels of protein tyrosine nitration (Fig. 6a) and similar protein carbonylation (Fig. 6b), indicating higher levels of NO-induced oxidative stress in the H-ALF. Confirming the low levels of ROSoxidation and no differences in protein carbonylation observed in 
a

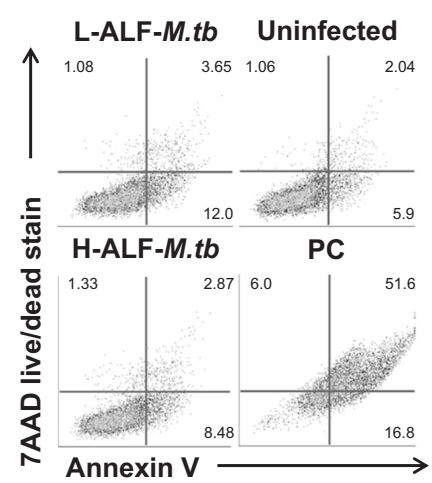

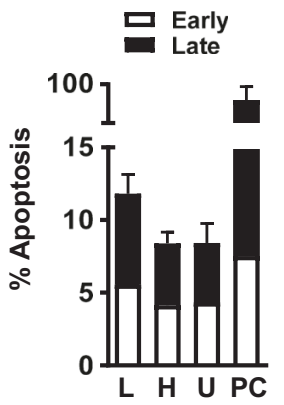

d
C

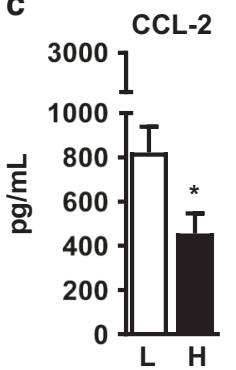

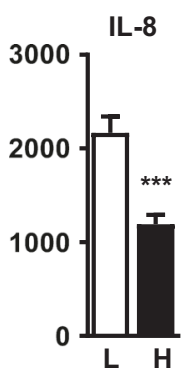
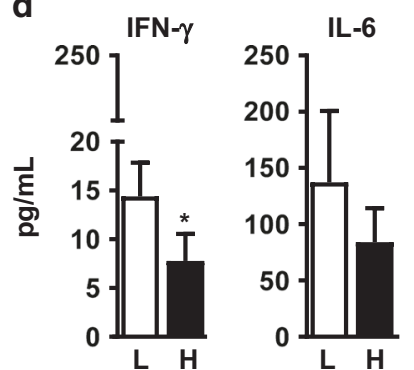

e
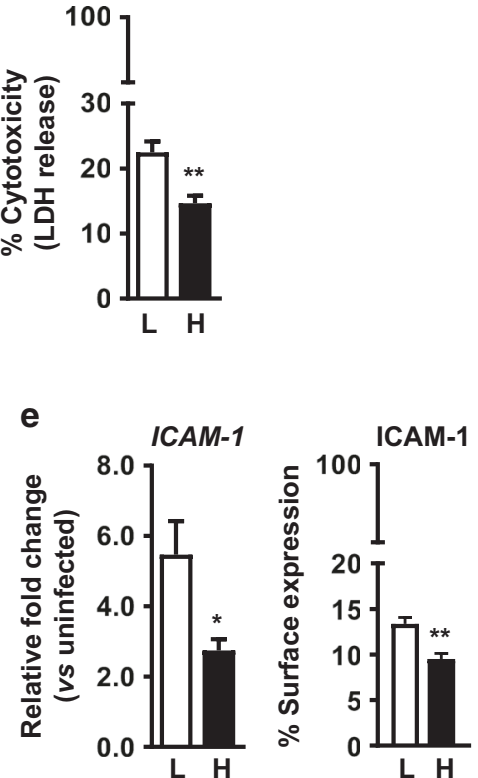

Fig. 4 H-ALF-M.tb induces less cell death and decreases AT immune mediators. ATs were infected with L-ALF and H-ALF-M.tb for 5 days for determination of AT cell death $(\mathbf{a}, \mathbf{b})$ or AT immune mediator production $(\mathbf{c}, \mathbf{d})$ and expression $(\mathbf{e}, \mathbf{f})$. a ATs were assessed for early and late apoptosis/necrosis by flow cytometry using Annexin V \& 7-AAD live/dead staining per kit instructions. Representative flow cytometry plots are shown on left and quantification on right. Experiment performed in duplicate (mean \pm SEM); $n=4$ Low ALFs \& 3 High ALFs. b AT supernatants were tested for $\%$ cytotoxicity by the release of LDH (measure of necrosis), per kit instructions; $n=7 \mathrm{~L}-\mathrm{ALFs} \& 5 \mathrm{H}-\mathrm{ALFs}$ in duplicate (mean \pm SEM). c AT chemokine production in cell supernatants measured by ELISA per kit instructions. $N=4 \mathrm{~L}-\mathrm{ALFs}$ and $4 \mathrm{H}$-ALFs in triplicate; mean \pm SEM. d AT cytokine production in cell supernatants tested by Multiplex Assay per kit instructions. $N=4 \mathrm{~L}-\mathrm{ALFs}$ and $4 \mathrm{H}$-ALFs in duplicate; mean \pm SD. e (Left) AT mRNA expression measured by qRT-PCR and shown as relative fold change versus uninfected ATs. $N=3$ Low and 3 High ALFs, in duplicate; mean \pm SEM. (Right) Percentage of AT cell surface expression of ICAM-1 assessed by flow cytometry. $N=2$ Low and 2 High ALFs, in replicate; mean \pm SEM. $\mathrm{L}=\mathrm{L}$-ALF-M.tb, $\mathrm{H}=\mathrm{H}-\mathrm{ALF}-M . t b, \mathrm{U}=$ uninfected ATs \& PC = ethanol-treated ATs. Student's $t$-test, L-ALF- vs. HALF-M.tb; ${ }^{*} p<0.05,{ }^{* *} p<0.01,{ }^{* * *} p<0.001$

a

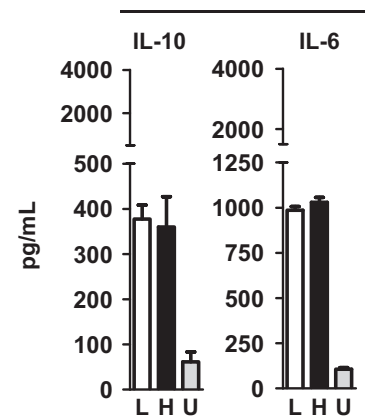

Direct contact

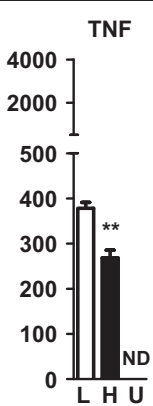

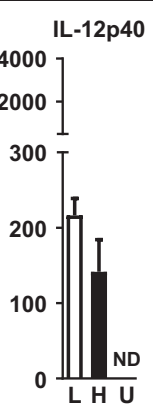

b

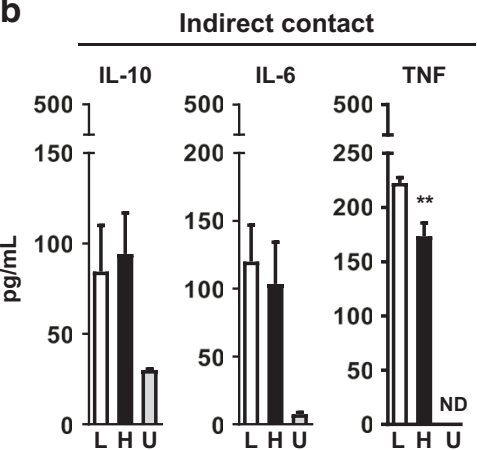

Fig. 5 H-ALF-M.tb infected ATs modulate human macrophage immune response. ATs were infected with L- or H-ALF-M.tb for 5 days and a exposed-M.tb infected ATs were co-cultured with primary human monocyte-derived macrophages (MDMs) or b exposed-M.tb infected AT supernatant was $0.2 \mu \mathrm{m}$-sterile filtered and incubated with MDMs. After $24 \mathrm{~h}$, macrophage protein production was assessed by ELISA per kit instructions to determine a direct and $\mathbf{b}$ indirect modulation of MDM cytokine release by exposed-M.tb ATs. $N=3$ Low and 3 High ALFs, performed in duplicate (mean \pm SEM) with 2 different MDM donors. L = resting MDMs incubated with L-ALF-M.tb infected ATs (or supernatant), $\mathrm{H}=$ resting MDMs incubated with H-ALF-M.tb infected ATs (or supernatant), $\mathrm{U}=$ resting MDMs incubated with uninfected ATs or (supernatant). Student's $t$-test, L-ALF- vs. H-ALF-M.tb; ${ }^{* *} p<0.01$. ND $=$ not determined

both ALF subsets, there were also low levels of MPO (a marker of ROS oxidation and neutrophil activity in the alveolar space ${ }^{19}$ ) and no differences between L- and H-ALF (Fig. $6 c$ ).

Increased protein tyrosine nitration in H-ALF may impact or reflect changes in protein function; therefore, we tested ALFinnate protein function as the ability of ALF-proteins to bind to the M.tb surface following exposure to L- and H-ALF. We focused on the M.tb-binding ability of three major innate proteins $(C 3, S P-A$ and SP-D) described to play a critical role in M.tb-host interactions. ${ }^{11,20-22}$ For all three proteins, we observed a correlation between increased H-ALF protein nitration and decreased innate protein function in H-ALF (Fig. $6 \mathrm{~d}-\mathrm{f}$ ). Through multivariate regression analysis of ALF status (i.e., L- vs. H-ALF), 3-nitrotyrosine content and innate protein levels against innate protein function 

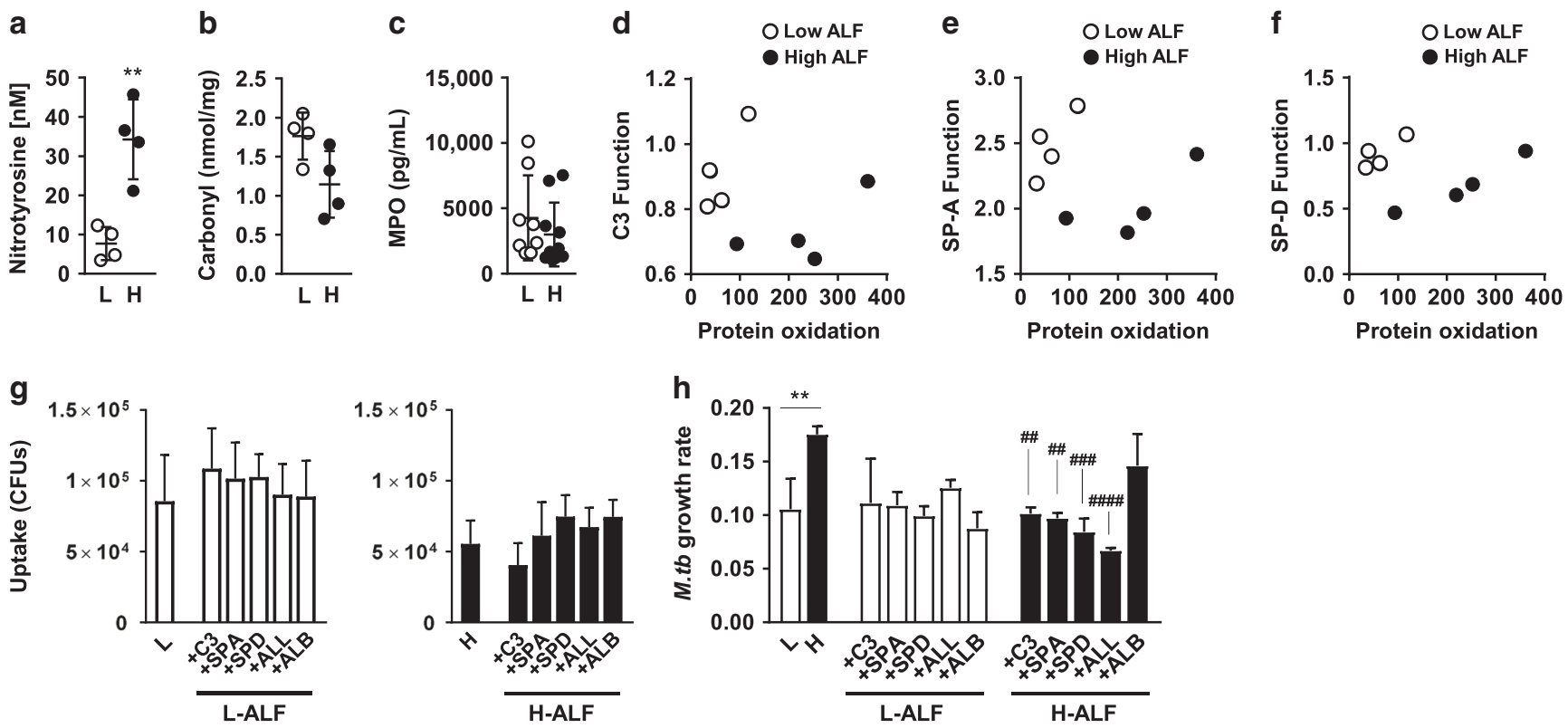

Fig. $6 \mathrm{H}$-ALF- proteins have increased tyrosine nitration, are less functional, and drive enhanced H-ALF exposed-M.tb growth rate in ATs. Levels of oxidative stress in Low- $(\mathrm{L})$ and High- $(\mathrm{H})$ ALF samples were detected by ELISA for the presence of a 3-nitrotyrosine residues, $\mathbf{b}$ carbonyls or c myeloperoxidase (MPO). All ALFs were run in duplicate for $n=4$, mean \pm SD. Student's $t$-test, L-ALF vs. H-ALF; ${ }^{* *} p<0.01$. Innate protein function, measured as the ability of innate proteins to bind to the M.tb surface, was determined using indirect ELISA after M.tb exposure to L- or H-ALF for $12 \mathrm{~h}(\mathbf{d}-\mathbf{f})$. Binding assays were performed in triplicate and calculated as the absorbance (OD $450 \mathrm{~nm}) \mathrm{vs}$. positive control (human SP-A protein $(3 \mu \mathrm{g})$, human SP-D protein $(1 \mu \mathrm{g})$ and human C3 protein $(10 \mu \mathrm{g})$. Innate protein function (y-axis) is plotted as a function of ALF subset (Low ALF, shown as white circles and High ALF, as black circles) and 3-nitrotyrosine content (x-axis) for d C3, e SP-A and f SP-D. Shown is $n=4$, mean \pm SD. All data shown has been normalized for $1 \mathrm{mg} / \mathrm{mL}$ ALF phospholipid content. $\mathbf{g}, \mathbf{h} M . t b$ was exposed to Low ALF, High ALF, or L-ALF and H-ALF replenished with the following recombinant human proteins for $12 \mathrm{~h}: \mathrm{C} 3(+\mathrm{C} 3,20 \mu \mathrm{g} / \mathrm{mL}), \mathrm{SP}-\mathrm{A}(+\mathrm{SP}-\mathrm{A}, 10$ $\mu \mathrm{g} / \mathrm{mL})$, SP-D + SP-D, $5 \mu \mathrm{g} / \mathrm{mL})$, all 3 combined ( + ALL), or human serum albumin $(40 \mu \mathrm{g} / \mathrm{mL})$ as an irrelevant protein control ( + ALB). Following the 12-hour incubation, exposed-M.tb were pelleted, washed to remove traces of ALF and unbound proteins, and used to infect ATs at MOI 10:1 for $2 \mathrm{~h}$. Low ALFs are shown in white bars and High ALFs shown in black bars. g AT-exposed-M.tb uptake was assessed after 2-hour infection and 1-hour gentamicin treatment for determination of intracellular CFUs. $\mathbf{h}$ Exposed-M.tb growth rate (slope of M.tb intracellular growth in ATs) was determined throughout 5 days of intracellular AT infection. Shown is $n=4$ Low and 4 High ALFs (ALFs without replenishment) and $n=2$ Low and 2 High ALFs for respective ALF-replenishment studies, mean \pm SEM. One-way ANOVA post-Dunnett's

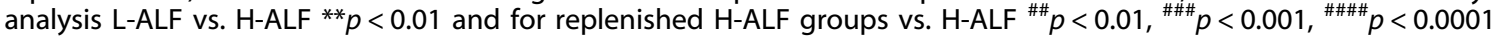

(Tables 1-3 and Tables S1-3), we observed a significant inverse correlation between C3 protein levels and C3 function in ALF ( $p=$ 0.045) (Table 1). In the case of SP-D both ALF status and protein tyrosine nitration correlated with SP-D function ( $p=0.001$ for ALF status; $p=0.009$ for protein 3-nitrotyrosine content) (Table 3). Also, for SP-A we observed significantly decreased SP-A binding to the M.tb surface in H-ALF $\left(\mathrm{OD}_{450} 1.915 \pm 0.1322\right.$ in $\mathrm{H}-\mathrm{ALF}$ vs. $\mathrm{OD}_{450}$ $2.361 \pm 0.1243$ in L-ALF, $p<0.05)$. Together, these results suggest that the oxidation status may contribute to H-ALF-innate proteins being less functional.

H-ALF replenishment with functional innate proteins reverses M.tb growth rate

We next chose to replenish $\mathrm{H}$-ALF with functional proteins prior to H-ALF-M.tb exposure to test its impact on M.tb growth rate in ATs. We incubated M.tb in H-ALF in the absence and presence of recombinant human proteins $\mathrm{C}$, SP-A, or SP-D (alone or in combination). Our results show H-ALF replenishment with any of these proteins did not alter M.tb uptake by ATs (Fig. 6g, black bars), supporting our previous findings that H-ALF- vs. L-ALF-M.tb growth rate is uptake-independent. However, H-ALF replenishment with C3, SP-A, and/or SP-D reversed the M.tb growth rate to the levels observed for L-ALF M.tb during AT intracellular infection (Fig. 6h, black bars). This observation was specific to the addition of C3, SP-A and/or SP-D to H-ALF, as no difference in uptake (Fig. 6g, white bars) and M.tb growth rate (Fig. 6h, white bars) were observed when L-ALF was replenished with these proteins and/or when the irrelevant protein albumin was added to L- and H-ALFs prior to M.tb exposure and AT infection (Fig. $6 \mathrm{~g}, \mathrm{~h},+A L B$ ). Together, these results suggest that dysfunctional innate proteins in H-ALF drive significantly enhanced M.tb growth rate in ATs.

\section{DISCUSSION}

Our premise is that M.tb deposited into the distal lung will encounter ALF, and interactions with innate soluble host factors and cells present in this environment will shape the outcome of infection. ${ }^{4-8}$ We have demonstrated an important role for the human ALF in altering the M.tb cell wall surface without compromising its viability and capability to replicate extracellularly. ${ }^{4-7}$ These ALF derived M.tb cell wall surface alterations subsequently dictate M.tb-phagocyte interactions. ${ }^{4-7}$ Indeed, several reports have demonstrated a role of ALF components in altered TB susceptibility, such as reports of polymorphisms in surfactant proteins $A$ and $D$ that have been identified by epidemiological studies in in Ethiopia, Mexico, and India. ${ }^{23-25}$ Thus, genetic evidences support ALF components are critical for $M . t b$ infection and progression to TB disease in humans.

Recently, increased interest has been given to the role of nonphagocytic cell populations in M.tb infection. ${ }^{26}$ In the case of M.tbinfected ATs, published evidence in vitro supports primarily a proinflammatory phenotype marked with cell necrosis and enhanced bacterial replication. ${ }^{17}$ This is in contrast to limited in vivo studies, including the identification of M.tb DNA within nongranulomatous tissue in individuals with latent TB infection suggesting ATs harbor M.tb in the absence of clinical disease. ${ }^{27}$ 
Table 1. Multivariate regression analysis of complement C3 function Regression Statistics

\begin{tabular}{ll}
\hline Multiple R & 0.941437406 \\
R Square & 0.88630439 \\
Adjusted R Square & 0.801032683 \\
Standard Error & 0.065027263 \\
Observations & 8 \\
Variable & $P$-value \\
ALF subset (L vs. H) & 0.251788855 \\
Oxidation & 0.297227165 \\
Innate protein levels & $0.045017272^{*}$
\end{tabular}

Multivariate regression analysis of ALF subset (Low vs. High), oxidation (total protein nitrosylation) and innate protein levels (concentration) vs. innate protein function (M.tb-binding) for $\mathrm{C} 3 ;{ }^{*} p<0.05$

Table 2. Multivariate regression analysis of surfactant protein A function

Regression Statistics

\begin{tabular}{ll}
\hline Multiple R & 0.909350813 \\
R Square & 0.826918901 \\
Adjusted R Square & 0.567297252 \\
Standard Error & 0.243153484 \\
Observations & 6 \\
Variable & $P$-value \\
ALF subset (L vs. H) & 0.103583238 \\
Oxidation & 0.254725881 \\
Innate protein levels & 0.930708418
\end{tabular}

Multivariate regression analysis of ALF subset (Low vs. High), oxidation (total protein nitrosylation) and innate protein levels (concentration) vs. innate protein function (M.tb-binding) for SP-A

Table 3. Multivariate regression analysis of surfactant protein $D$ function

Regression Statistics

\begin{tabular}{ll}
\hline Multiple R & 0.973692482 \\
R Square & 0.94807705 \\
Adjusted R Square & 0.909134838 \\
Standard Error & 0.059173673 \\
Observations & 8 \\
Variable & $P$-value \\
ALF subset (L vs H) & $0.001445561^{* *}$ \\
Oxidation & $0.008554315^{* *}$ \\
Innate protein levels & 0.251612266
\end{tabular}

Multivariate regression analysis of ALF subset (Low vs. High), oxidation (total protein nitrosylation) and innate protein levels (concentration) vs. innate protein function (M.tb-binding) for SP-D; ${ }^{* *} p<0.01$

As we have previously shown that the human lung mucosa dampens the inflammatory response and enhances bacterial control in M.tb-infected phagocytes, ${ }^{4-7}$ we chose to extend our studies to the role ATs in M.tb infection.

We chose to utilize the alveolar epithelial type II-like cell line A549, which allowed us to exclusively focus on heterogeneity of the lung mucosa by using 14 different healthy human ALF samples. We observed remarkable variability in the rate of M.tb intracellular growth in ATs, which allowed us to differentiate ALFs into two subtypes: the ones that allow ATs to better control M.tb intracellular growth and those that promote increased M.tb growth. This differential growth rate was independent of the levels of uptake observed, with an average of less than $5 \%$ of the bacteria in the inoculum being internalized by ATs. Low frequency of uptake during M.tb-AT infection has been reported. ${ }^{28}$ This low frequency of contact between M.tb and ATs, in comparison to $M$. tb-phagocyte interactions, is a more likely scenario and allows for us to closely examine minute differences that drive the differential growth rate observed in ATs.

Our previous studies using professional phagocytes indicate that ALF exposed-M.tb is always cleared better. ${ }^{4-7}$ This is in contrast to our results presented here, which suggests that ALF influences M.tb infection in a cell type-specific manner. In support of this, several respiratory pathogens, including M.tb, have been shown to induce different responses in vitro during AT and macrophage infection rendering certain cell populations more permissive to bacterial growth. ${ }^{29,30}$ Due to the predominance of ATs in the lung space, ${ }^{31}$ M.tb may first contact ATs during initial stages of infection. It is therefore likely that the response of the first cell contacted in the lung environment during infection may be critical for the outcome of M.tb infection.

Here we show that ALF drives differential M.tb growth rate, independent of uptake, which prompted us to explore AT intracellular trafficking pathways. We did not detect changes in autophagy, the endosomal trafficking or lipid transport pathways; however, we found that M.tb exposed to the H-ALF subset had significantly enhanced active replication during AT infection. Moreover, of the H-ALF-M.tb undergoing active replication we observed greater co-localization within AT MVBs and LAMP-1+ compartments. ${ }^{4-7}$ MVBs have been shown by others to be utilized for pathogen survival and replication due to their rich lipid and cholesterol composition. ${ }^{32,33}$ As MVBs are capable of preventing lysosomal degradation of cargo, either transiently or through recycling mechanisms, ${ }^{34}$ use of this AT compartment would be beneficial to M.tb for growth. MVBs have been also shown to contain LAMPs, ${ }^{34}$ and thus our results cannot rule out the intersection of these two pathways during M.tb infection. Thus, the sum of H-ALF-induced modifications and the AT intracellular trafficking pathway utilized by the bacterium may be the perfect combination for the enhanced replication observed.

The fact that H-ALF exposed M.tb infected ATs had decreased CCL-2 production and ICAM-1 expression suggested that interactions between ATs and macrophages could be altered. Published reports show ATs alter phagocyte activation through direct and indirect mechanisms. ${ }^{17,35-40}$ We observed, relative to M.tb exposed to L-ALF, H-ALF-M.tb infected ATs induced lower levels of pro-inflammatory cytokines produced by macrophages both through direct and indirect mechanisms. Our similar findings in these two experimental setups confirm that the source of cytokines in direct studies is macrophages. We also observed that ATs do not produce detectable levels for the cytokines tested (data not shown). Others have reported that factors secreted from stimulated ATs influence macrophage phagocytosis; ${ }^{36}$ but in our case, we did not detect changes in macrophage phagocytosis in response to soluble factors from L- and H-ALF-M.tb infected ATs (data not shown).

ALF-containing innate proteins are critical in the immune response to $M . t b$ infection. ${ }^{2,41,42}$ Imbalances in the oxidation status of ALF can alter ALF protein function. ${ }^{43}$ We observed that H-ALF proteins have enhanced tyrosine nitration, in comparison to L-ALF proteins. Additionally, we found that H-ALF proteins were less functional in their ability to bind to the M.tb surface. Modification of tyrosine residues in $\mathrm{H}$-ALF proteins to 3-nitrotyrosine by peroxynitrite or other nitrating agents is indicative of oxidative 
stress in biological systems. ${ }^{44}$ Currently, we cannot definitively state that H-ALF innate proteins (i.e., C3, SP-A, and SP-D) have enhanced tyrosine nitration; however, this is an ongoing line of research in our lab. When replenishing the function of C3, SP-A and SP-D in H-ALF using their estimated physiological ranges, ${ }^{45-48}$ the high M.tb growth rate over 5 days of intracellular AT infection decreased to the levels observed for L-ALF. This indicates a redundant function for these proteins during M.tb infection of ATs and/or a bacterial-mediated growth effect, in which M.tb exposure to functional innate proteins increases host-induced stress on $M$. $t b$. Thus, ALF-innate proteins may act in a bacteriostatic manner to slow M.tb replication in ATs. Similar results obtained with all innate proteins tested may be due to compensatory pathways within ATs. Importantly, replenishing Low ALF with innate proteins prior to M.tb exposure did not change M.tb uptake and growth rate within ATs, supporting that the observed mechanism is specific to $\mathrm{H}-\mathrm{ALF}$ and not a general response to adding antimicrobial proteins to ALF. Ongoing studies will address M.tb transcriptional and metabolic changes in response to L- and H-ALF exposure.

In summary, our studies presented here add to our previous findings in demonstrating the importance of accounting for both lung microenvironment and the first cell to contact M.tb in the alveolar space, as these factors together may drive M.tb infection and influence TB disease outcome. In this regard, we should consider the status of the human lung mucosa in promoting M.tb replication within ATs while dampening cell migration into the lung, which may provide enough time and a place for M.tb to establish a successful infection, as well as consider ALF soluble components as host-directed therapeutic targets to enhance early M.tb control.

\section{METHODS}

\section{Ethics statement}

This study was carried out in strict accordance with US Code of Federal and Local Regulations (The Ohio State University IRB numbers 2008H0135 \& 2008H0119 and Texas Biomedical Research Institute/UT-Health San Antonio IRB number HSC20170667H), and Good Clinical Practice as approved by the National Institutes of Health (NIAID/DMID branch) with written informed consent from all subjects.

\section{AT culture}

The alveolar epithelial type II-like cell line A549 was maintained at $37^{\circ} \mathrm{C}$ with $5 \% \mathrm{CO}_{2}$ in DMEM/F12 (ThermoFisher Scientific, Waltham, MA) supplemented with 10\% FBS (Atlas Biologicals, Fort Collins, CO) and 10,000 U/mL PenStrep (Sigma, St. Louis, MO). Cells were maintained in antibiotic-free growth medium at least $24 \mathrm{~h}$ prior to use in experiments. For multiplicity of infection (MOI), 3 wells were used to determine cell count. All experiments were conducted at MOI 10:1.

\section{M.tb culture}

GFP-M.tb Erdman (kindly provided by Dr. Marcus Horwitz, UCLA) was grown as we previously described. ${ }^{7}$ SSB-GFP, smyc'::mCherry M.tb Erdman (kindly provided by Dr. David Russell, Cornell University) was grown as described. ${ }^{14}$

Human ALF isolation

ALF was obtained from human bronchoalveolar lavage fluid (BALF) and was normalized as we described ${ }^{4-8}$ from healthy human donors. See Supplementary material for details.

\section{Preparation of ALF exposed-M.tb}

Exposure of M.tb to human ALF was performed as we published. ${ }^{4-}$

8 Briefly, M.tb single cell suspension $\left(1 \times 10^{8}\right)$ was achieved, followed by exposure to human ALF $(50 \mu \mathrm{L})$ at its physiological concentration within the human lung, or left unexposed as a control (unexposed-M.tb).for $12 \mathrm{~h}$ at $37^{\circ} \mathrm{C}, 5 \% \mathrm{CO}_{2}$. Following this exposure M.tb was washed several times to remove all ALF and resuspended in sterile $0.9 \% \mathrm{NaCl}$. Exposed-M.tb was used immediately. For determination of extracellular M.tb growth (viability), ALF-exposed- or unexposed-M.tb was serially diluted in 7H9 broth and plated on 7H11 agar for determination of M.tb extracellular growth by colony forming units (CFUs). In all experiments, inoculums were also platted confirming M.tb viability and no differences in viable bacterial counts among them.

\section{M.tb infection of Ats}

ATs were infected for $2 \mathrm{~h}, 37^{\circ} \mathrm{C}, 5 \% \mathrm{CO}_{2}$, with the first $30 \mathrm{~min}$ of infection performed shaking. After infection, unbound bacteria were removed by washing and gentamicin $(50 \mu \mathrm{g} / \mathrm{mL})$-supplemented medium was added for $1 \mathrm{~h}$. Cells were used immediately or returned to the incubator with $10 \mu \mathrm{g} / \mathrm{mL}$ gentamicinsupplemented growth medium until indicated times. For all experiments, ' $n$ ' values correspond to the number of different ALFs used for M.tb exposure. Each ALF sample is from a different healthy human donor.

\section{AT uptake of ALF exposed-M.tb}

AT uptake of M.tb was determined following exposed-M.tb AT infection. Wells were lysed with cold $0.1 \%$ Triton-X100 in PBS for $15 \mathrm{~min}$. Cell lysates were serially diluted in $7 \mathrm{H} 9$, plated, and cultured on $7 \mathrm{H} 11$ agar plates at $37^{\circ} \mathrm{C}$ for up to 21 days.

Intracellular growth of ALF exposed-M.tb in ATs ATs were infected with exposed-M.tb and intracellular bacterial growth was determined at $5 \mathrm{DPI}$. The supernatant was centrifuged for $5 \mathrm{~min}$ at $300 \times g 4^{\circ} \mathrm{C}$, and cells were added to respective wells for lysis. Monolayers were lysed with cold $0.1 \%$ Triton-X100 in PBS for $15 \mathrm{~min}$. Cell lysates were serially diluted in $7 \mathrm{H} 9$, plated, and cultured on $7 \mathrm{H} 11$ agar plates at $37^{\circ} \mathrm{C}$ for up to 21 days.

Acidification of ALF exposed-M.tb containing intracellular compartments in Ats

At indicated times post-infection, M.tb-compartment acidification was determined as we previously published. ${ }^{4,6}$ Refer to Supplementary Material.

\section{Intracellular trafficking of ALF exposed-M.tb in Ats}

At indicated times post-infection, ATs were fixed with cold $4 \%$ paraformaldehyde for $15 \mathrm{~min}$ at room temperature and permeabilized with $0.1 \%$ Triton-X100 for $10 \mathrm{~min}$ at room temperature. To assess exposed M.tb intracellular trafficking, compartments were stained with primary antibodies (overnight incubation at $4{ }^{\circ} \mathrm{C}$ ) and secondary antibodies $\left(60 \mathrm{~min}\right.$ incubation at $\left.37^{\circ} \mathrm{C}\right)$ or matched isotype controls. Co-localization was quantified by counting $\geq 150$ events per coverslip, performed in replicate using the Olympus FV1000 Filter Confocal Microscope and Zeiss LSM 800 Confocal Microscope. All microscopy data were analyzed with the Olympus FluoView Viewer and Zeiss ZEN Software. For specific antibody information, refer to Supplementary Material.

Early and late apoptosis of ALF exposed-M.tb infected ATs by flow cytometry

At 5 DPI early (Annexin $\mathrm{V}^{+}$) and late apoptosis/necrosis (Annexin $\mathrm{V}^{+} 7 \mathrm{AAD}^{+}$) of exposed-M.tb infected ATs was determined using PE-Annexin V Apoptosis \& 7AAD Live/Dead Stain Detection Kit (BD Biosciences) as we described. ${ }^{5,6}$ Cells were analyzed using a BD LSRII flow cytometer (BD Biosciences, San Jose, CA). At least 10,000 events were counted and analyzed using FlowJo Version 9.7.6 Software.

\section{ATs $L D H$ release}

AT cytotoxicity (measured by LDH release as a measure of cell necrosis ${ }^{49}$ ) from exposed-M.tb infected ATs was determined by 
colorimetric assay per kit instructions (CytoTox 96 ${ }^{\circledR}$ NonRadioactive Cytotoxicity Assay kit, Promega, Madison, WI).

Protein production by ALF-M.tb infected ATs

Protein levels in supernatants from exposed-M.tb infected ATs at 5 DPI were determined using: (1) Human enzyme-linked immunosorbent assay (ELISA) kits for TNF, IL-6, IL-8, CCL2, IL-1 $\beta$, IL-12p40, and IL-10 (R\&D Systems, Minneapolis, MN) and TGF- $\beta$ and IFN- $\gamma$ (BD Biosciences, San Jose, CA) and (2) Human $\mathrm{TH}_{1} / \mathrm{TH}_{2}$ Cytokine ProcartaPlex Panel (ThermoFisher Scientific, Waltham, MA), per kit instructions. ProcartaPlex analysis was performed using MasterPlex (Hitachi Solutions America) version 2.0.0.80 software.

RNA isolation and gene expression

Following 5 days exposed-M.tb infection, AT gene expression was determined as we previously described. ${ }^{5,6}$ Refer to Supplementary Material.

L- vs. H-ALF exposed-M.tb modulation of AT surface marker expression

After 5 days of exposed-M.tb infection, ATs were stained with antibodies specific for cell surface markers, or matched isotype controls. AT surface expression was assessed by counting $\geq 10,000$ events. Samples were read on a BD LSRII flow cytometer (BD Biosciences) and data were analyzed using FlowJo Version 9.7.6 Software. For specific antibody information, refer to Supplementary Material.

Preparation of human monocyte-derived macrophages (MDMs) Human MDMs were obtained from peripheral blood mononuclear cells (PBMCs) from healthy, tuberculin-negative donors as we previously described. ${ }^{4-7}$ In some experiments, PBMCs on day $5 / 6$ of culture were used.

L- vs. H-ALF exposed-M.tb infected AT modulation of resting macrophage immune response

Supernatants from 5 DPI exposed-M.tb infected ATs were $0.2 \mu \mathrm{m}$ filtered and added to resting day 5/6 MDMs (indirect contact) or exposed-M.tb infected AT monolayers were co-cultured with resting MDMs (direct contact) for $24 \mathrm{~h}$. After $24 \mathrm{~h}$, supernatants were collected, $0.2 \mu \mathrm{m}$-filtered and protein content was determined by ELISA (R\&D Systems) according to the manufacturer's instructions.

L- vs. H-ALF-M.tb infected AT modulation of resting macrophage activation marker expression

After 5 days exposed-M.tb infection, AT supernatants were $0.2 \mu \mathrm{m}$ filtered and incubated with resting PBMCs for $24 \mathrm{~h}$ at $37^{\circ} \mathrm{C}, 5 \%$ $\mathrm{CO}_{2}$. PBMCs were stained with antibodies specific for cell surface markers, or matched isotype controls. Macrophage (gated CD $11 \mathrm{c}^{+}$ population) activation marker surface expression was assessed by flow cytometry by counting $\geq 10,000$ events. Samples were read on a BD LSRII flow cytometer (BD Biosciences) and data were analyzed using FlowJo Version 9.7.6 Software. For specific antibody information, refer to Supplementary Material.

Levels of ALF-containing immune mediators

Protein levels in human ALF were determined using custom Human Magnetic Luminex Assays (R\&D Systems), Human $\mathrm{TH}_{1} / \mathrm{TH}_{2}$ Cytokine ProcartaPlex Panel (ThermoFisher Scientific), and by Human SP-A, SP-D and C3 ELISA (LifeSpan Biosciences, Inc., Seattle, WA) per kit instructions.

Determination of low vs. high ALF protein tyrosine nitration Levels of 3-Nitrotyrosine-containing proteins (stable marker of RNS-induced oxidation) in human ALF (using $10 \mu \mathrm{g}$ protein, per kit instructions) were determined using OxiSelect Nitrotyrosine ELISA kit (Cell Biolabs, Inc., San Diego, CA).
Determination of Low vs. High ALF protein carbonylation Detection of protein carbonyls (stable marker of ROS-induced oxidation) in ALF (using $8 \mu \mathrm{g}$ protein, per kit instructions) was determined using OxiSelect Protein Carbonyl ELISA Kit (Cell Biolabs, Inc.).

Determination of MPO in ALF

MPO levels in human ALF were determined using human MPO quantikine ELISA kit (R\&D Systems).

H- vs. L-ALF innate protein-M.tb binding activity assays

Following M.tb exposure to human ALF, exposed-M.tb was washed to remove all traces of ALF and resuspended in $500 \mu \mathrm{L}$ of TBS +2 $\mathrm{mM} \mathrm{CaCl}$ (TBS-C). M.tb $\left(1 \times 10^{7}\right)$ was added to triplicate wells of 96-well plates and dried overnight. Binding of SP-A, SP-D, and C3 to exposed-M.tb was determined by indirect ELISA using primary antibodies (mouse anti-human SP-A, mouse anti-human SP-D and mouse anti-human $\mathrm{C} 3 / \mathrm{C} 3 \mathrm{~b}$; Abcam) and secondary antibodies (donkey anti-mouse HRP and goat-anti-rabbit HRP; Santa Cruz). Absorbance was read at $450 \mathrm{~nm}$ using a GloMax plate reader (Version 3.0; Promega Corporation). Protein binding was plotted as absorbance relative to positive control.

ALF-innate protein replenishment and exposed-M.tb infection of ATs

At the time of M.tb exposure to human ALF, L-ALF, or H-ALF samples were replenished with the following recombinant human proteins: C3 $(20 \mu \mathrm{g} / \mathrm{mL}$, Complementech, Tyler, Texas), SP-A (10 $\mu \mathrm{g} /$ $\mathrm{mL}$, patient-purified), SP-D $(5 \mu \mathrm{g} / \mathrm{mL}, R \& D$ Systems), or all 3 combined at the concentrations described. As an irrelevant protein control, L-ALF and H-ALF were replenished with human serum albumin $(40 \mu \mathrm{g} / \mathrm{mL}$, CSL Behring, King of Prussia, PA). M.tb exposure to replenished ALF was performed as described above. ${ }^{4-8}$

Statistical analyses

GraphPad 5.0 \& 7.0 Prism software was used for statistical analyses to perform an unpaired, 2-tailed Student $t$-test of the difference between two means and to perform a One-way ANOVA for multiple groups comparison, followed by post-Tukey and postDunnett's analyses. Microsoft Excel Data Analysis Toolpak was used to perform Multivariate Regression Analysis to determine correlations among the variables of interest, i.e., ALF status, protein tyrosine nitration (measured by 3-nitrotyrosine content), and innate protein levels, vs. protein function (binding determined by $\left.\mathrm{OD}_{450}\right)$. For regression analyses, all data were normalized to 1 mg phospholipid per ml ALF.

\section{ACKNOWLEDGEMENTS}

This work was supported by the NIH/NIAID (AI073856 \& AI093570 to JBT and CMIB T32-Al112542 to JMS) and The Ohio State University (OSU) College of Medicine Systems in Integrative Biology Training Program Fellowship supported by the $\mathrm{NIH}$ / NIGMS (SIB T32-GM068412 grant to JMS). This work was also supported by the Forum Grant to JBT at the Texas Biomedical Research Institute. We thank Dr. David Russell and his laboratory personnel for kindly providing us with the SSB-GFP M.tb Erdman reporter strain. We also thank Mr. Russell Ault for his assistance in our statistical analyses and valuable comments, as well as the Biosafety Level-3 Programs at Texas Biomedical Research Institute and OSU for their services and support.

\section{AUTHOR CONTRIBUTIONS}

J.M.S., A.O.F., H.V.K., S.S., J.A., A.A., M.D.W., and J.B.T. had substantial contributions to the conception or design of the work; or the acquisition, analysis, and/or interpretation of data. J.M.S. and J.B.T. drafted the work and/or revised it critically for important intellectual content. 


\section{ADDITIONAL INFORMATION}

The online version of this article (https://doi.org/10.1038/s41385-019-0156-2) contains supplementary material, which is available to authorized users.

Competing interests: The authors declare no competing interests.

Publisher's note: Springer Nature remains neutral with regard to jurisdictional claims in published maps and institutional affiliations.

\section{REFERENCES}

1. Erck, D. Take $1.5 \mathrm{~min}$ for 1.5 million lives. (Aeras, 2016). www.aeras.org

2. Torrelles, J. B. \& Schlesinger, L. S. Integrating lung physiology, immunology, and tuberculosis. Trends Microbiol 25, 688-697 (2017).

3. Sia, J. K., Georgieva, M. \& Rengarajan, J. Innate immune defenses in human tuberculosis: An overview of the interactions between Mycobacterium tuberculosis and innate immune cells. J. Immunol. Res. 2015, 12 (2015).

4. Arcos, J. et al. Mycobacterium tuberculosis cell wall released fragments by the action of the human lung mucosa modulate macrophages to control infection in an IL-10-dependent manner. Mucosal Immunol. 10, 1248-1258 (2017).

5. Scordo, J. M. et al. Mycobacterium tuberculosis cell wall fragments released upon bacterial contact with the human lung mucosa alter the neutrophil response to infection. Front Immunol. 8, 307 (2017).

6. Arcos, J. et al. Lung mucosa lining fluid modifies Mycobacterium tuberculosis to reprogram human neutrophil killing mechanisms. J. Infect. Dis. 212, 948-958 (2015).

7. Arcos, J. et al. Human lung hydrolases delineate Mycobacterium tuberculosismacrophage interactions and the capacity to control infection. J. Immunol. 187, 372-381 (2011).

8. Moliva, J. I. et al. Exposure to human alveolar lining fluid enhances Mycobacterium bovis BCG vaccine efficacy against Mycobacterium tuberculosis infection in a CD8 (+) T-cell-dependent manner. Mucosal Immunol. 11, 968-978 (2018).

9. Fronius, M., Clauss, W. G. \& Althaus, M. Why do we have to move fluid to be able to breathe? Front Physiol. 3, 146 (2012).

10. Notter, R. H. Lung surfactants: Basic science and clinical applications. 1-444 (Marcel Dekker, New York, 2000).

11. Torrelles, J. B., Azad, A. K., Henning, L. N., Carlson, T. K. \& Schlesinger, L. S. Role of C-type lectins in mycobacterial infections. Curr. Drug Targets 9, 102-112 (2008).

12. Mvubu, N. E., Pillay, B., Gamieldien, J., Bishai, W. \& Pillay, M. Canonical pathways, networks and transcriptional factor regulation by clinical strains of Mycobacterium tuberculosis in pulmonary alveolar epithelial cells. Tuberc. (Edinb.) 97, 73-85 (2016).

13. Chai, A. B., Ammit, A. J. \& Gelissen, I. C. Examining the role of ABC lipid transporters in pulmonary lipid homeostasis and inflammation. Respir. Res 18, 41 (2017).

14. Sukumar, N., Tan, S., Aldridge, B. B. \& Russell, D. G. Exploitation of Mycobacterium tuberculosis reporter strains to probe the impact of vaccination at sites of infection. PLoS Pathog. 10, e1004394 (2014).

15. Dobos, K. M., Spotts, E. A., Quinn, F. D. \& King, C. H. Necrosis of lung epithelial cells during infection with Mycobacterium tuberculosis is preceded by cell permeation. Infect. Immun. 68, 6300-6310 (2000).

16. McDonough, K. A. \& Kress, Y. Cytotoxicity for lung epithelial cells is a virulenceassociated phenotype of Mycobacterium tuberculosis. Infect. Immun. 63, 4802-4811 (1995).

17. Scordo, J. M., Knoell, D. L. \& Torrelles, J. B. Alveolar epithelial cells in Mycobacterium tuberculosis Infection: Active players or innocent bystanders? J. Innate. Immun. 8, 3-14 (2016).

18. Sharma, M., Sharma, S., Roy, S., Varma, S. \& Bose, M. Pulmonary epithelial cells are a source of interferon-gamma in response to Mycobacterium tuberculosis infection. Immunol. Cell Biol. 85, 229-237 (2007).

19. Schmekel, B. et al. Myeloperoxidase in human lung lavage. I. A marker of local neutrophil activity. Inflammation 14, 447-454 (1990).

20. Schlesinger, L. S. Mycobacterium tuberculosis and the complement system. Trends Microbiol 6, 47-49 (1998).

21. Lugo-Villarino, G., Hudrisier, D., Tanne, A. \& Neyrolles, O. C-type lectins with a sweet spot for Mycobacterium tuberculosis. Eur. J. Microbiol Immunol. (Bp) 1, 25-40 (2011).

22. Ferguson, J. S. \& Schlesinger, L. S. Pulmonary surfactant in innate immunity and the pathogenesis of tuberculosis. Tuber. Lung Dis. 80, 173-184 (2000).

23. Azad, A. K., Sadee, W. \& Schlesinger, L. S. Innate immune gene polymorphisms in tuberculosis. Infect. Immun. 80, 3343-3359 (2012).
24. Singh, D. P., Bagam, P., Sahoo, M. K. \& Batra, S. Immune-related gene polymorphisms in pulmonary diseases. Toxicology 383, 24-39 (2017).

25. Goyal, S., Klassert, T. E. \& Slevogt, H. C-type lectin receptors in tuberculosis: What we know. Med Microbiol Immunol. 205, 513-535 (2016).

26. Randall, P. J., Hsu, N. J., Quesniaux, V., Ryffel, B. \& Jacobs, M. Mycobacterium tuberculosis infection of the 'non-classical immune cell'. Immunol. Cell Biol. 93, 789-795 (2015).

27. Hernandez-Pando, R. et al. Persistence of DNA from Mycobacterium tuberculosis in superficially normal lung tissue during latent infection. Lancet 356, 2133-2138 (2000).

28. Bermudez, L. E. \& Goodman, J. Mycobacterium tuberculosis invades and replicates within type II alveolar cells. Infect. Immun. 64, 1400-1406 (1996).

29. Coburn, J. \& Frank, D. W. Macrophages and epithelial cells respond differently to the Pseudomonas aeruginosa type III secretion system. Infect. Immun. 67, 3151-3154 (1999).

30. Huang, L., Nazarova, E. V., Tan, S., Liu, Y. \& Russell, D. G. Growth of Mycobacterium tuberculosis in vivo segregates with host macrophage metabolism and ontogeny. J. Exp. Med 215, 1135-1152 (2018).

31. Crabbé, A. et al. Alveolar epithelium protects macrophages from quorum sensing-induced cytotoxicity in a three-dimensional co-culture model. Cell Microbiol 13, 469-481 (2011).

32. Beatty, W. L. Trafficking from CD63-positive late endocytic multivesicular bodies is essential for intracellular development of Chlamydia trachomatis. J. Cell Sci. 119, 350-359 (2006)

33. Karjalainen, M. et al. Echovirus 1 infection depends on biogenesis of novel multivesicular bodies. Cell Microbiol 13, 1975-1995 (2011).

34. Piper, R. C. \& Katzmann, D. J. Biogenesis and function of multivesicular bodies. Annu Rev. Cell Dev. Biol. 23, 519-547 (2007).

35. Fehrenbach, $\mathrm{H}$. Alveolar epithelial type II cell: defender of the alveolus revisited. Respir. Res 2, 33-46 (2001).

36. Chuquimia, O. D., Petursdottir, D. H., Periolo, N. \& Fernandez, C. Alveolar epithelial cells are critical in protection of the respiratory tract by secretion of factors able to modulate the activity of pulmonary macrophages and directly control bacterial growth. Infect. Immun. 81, 381-389 (2013).

37. Chuquimia, O. D. et al. The role of alveolar epithelial cells in initiating and shaping pulmonary immune responses: communication between innate and adaptive immune systems. PLoS ONE 7, e32125 (2012).

38. Petursdottir, D. H., Chuquimia, O. D., Freidl, R. \& Fernandez, C. Macrophage control of phagocytosed mycobacteria is increased by factors secreted by alveolar epithelial cells through nitric oxide independent mechanisms. PLOS ONE 9, e103411 (2014)

39. Koyama, S. et al. Alveolar type II-like cells release G-CSF as neutrophil chemotactic activity. Am. J. Physiol. 275, L687-L693 (1998)

40. Sato, K. et al. Type II alveolar cells play roles in macrophage-mediated host innate resistance to pulmonary mycobacterial infections by producing proinflammatory cytokines. J. Infect. Dis. 185, 1139-1147 (2002).

41. King, R. J. Pulmonary surfactant. J. Appl. Physiol. 53, 1-8 (1982).

42. Weaver, T. E. \& Whitsett, J. A. Function and regulation of expression of pulmonary surfactant-associated proteins. Biochem. J. 273(Pt 2), 249-264 (1991).

43. Bargagli, E. et al. Oxidative stress in the pathogenesis of diffuse lung diseases: a review. Respir. Med 103, 1245-1256 (2009).

44. Gow, A. J., Farkouh, C. R., Munson, D. A., Posencheg, M. A. \& Ischiropoulos, H. Biological significance of nitric oxide-mediated protein modifications. Am. J. Physiol. Lung Cell Mol. Physiol. 287, L262-L268 (2004).

45. Wu, $\mathrm{H}$. et al. Surfactant proteins $A$ and $D$ inhibit the growth of Gram-negative bacteria by increasing membrane permeability. J. Clin. Invest 111, 1589-1602 (2003).

46. Ferguson, J. S., Weis, J. J., Martin, J. L. \& Schlesinger, L. S. Complement protein C3 binding to Mycobacterium tuberculosis is initiated by the classical pathway in human bronchoalveolar lavage fluid. Infect. Immun. 72, 2564-2573 (2004).

47. Gaynor, C. D., McCormack, F. X., Voelker, D. R., McGowan, S. E. \& Schlesinger, L. S. Pulmonary surfactant protein A mediates enhanced phagocytosis of Mycobacterium tuberculosis by a direct interaction with human macrophages. J. Immunol. 155, 5343-5351 (1995).

48. Ferguson, J. S., Voelker, D. R., McCormack, F. X. \& Schlesinger, L. S. Surfactant protein $\mathrm{D}$ binds to Mycobacterium tuberculosis bacili and lipoarrabinomannan via carbohydrate-lectin interactions resulting in reduced phagocytosis of the bacteria by macrophages. J. Immunol. 163, 312-321 (1999).

49. Chan, F. K., Moriwaki, K. \& De Rosa, M. J. Detection of necrosis by release of lactate dehydrogenase activity. Methods Mol. Biol. 979, 65-70 (2013). 